UDC: $811.163 .41^{\prime} 42$

DOI: https://doi.org/10.18485/beoiber.2018.2.1.4

\author{
Dragana Bajić ${ }^{1}$ \\ Instituto Cervantes de Belgrado \\ Serbia
}

\title{
MARCADORES DISCURSIVOS DE DISTANCIAMIENTO
}

\begin{abstract}
Resumen
Los estudios del discurso en la serbística actual están bastante marginados, a diferencia de los de los hispanistas. Uno de los temas especialmente tratados desde los comienzos del desarrollo de la pragmática en los ámbitos anglófono e hispano es el de los marcadores discursivos. Para esta ocasión hemos elegido un pequeño conjunto de ellos, los marcadores de distanciamiento, que tipológicamente pertenecen al grupo más amplio de los reformuladores. Como principales herramientas teóricas nos servirán las contribuciones de la filosofía del lenguaje, la cognitivista Teoría de la Pertinencia y la Teoría de la Argumentación con sus teorías coadyuvantes de los Topoi y la Polifónica. Una vez establecida la terminología, las definiciones y la clasificación, nos centraremos en el análisis contrastivo con un enfoque cualitativo. Teniendo en cuenta que la función de los marcadores es la de guiar las inferencias en el proceso de comunicación, buscaremos las equivalencias instruccionales de cada uno de los marcadores de distanciamiento con sus semejanzas y divergencias. El objetivo es determinar primero las unidades en serbio que se corresponden con las del español y luego establecer las condiciones y los tipos de correspondencia entre ellas. Los resultados obtenidos echarán luz también sobre el problema que surge de la diferencia entre los cuantificadores que forman parte de los marcadores y que puede afectar a la enseñanza y la adquisición de ambas lenguas por un lado, y a la traducción en ambos sentidos por el otro.
\end{abstract}

Palabras clave: marcador discursivo, reformulación, distanciamiento o separación, cuantificador.

\section{DISCOURSE MARKERS OF DISTANCING}

\begin{abstract}
Discourse research in the current Serbian studies is quite sidelined in comparison to Hispanic studies. From the onset of pragmatics in English and Hispanic studies, the discourse markers have received special attention. On this occasion we have chosen a small set of them, the markers of distancing or separation, which typologically belong to a wider group of reformulators. Our theoretical tools will rely on the contributions of the Philosophy of Language, the Theory of Relevance, Theory of Argumentation with its supporting theories of Topoi and Polyphony. Once we have established the terminology, definitions and

1 dacabaj@gmail.com
\end{abstract}

BEOIBERÍSTICA Vol. II / Número 1 (2018) | 57-72 
classification, we will focus on contrastive analysis with the qualitative approach. Bearing in mind that the purpose of the markers is to guide the inferences in the communicative process, we will search for instructional equivalents for each of the reformulators of distancing with its similarities and divergences. The aim is first to determine the units in Serbian which correspond to those in Spanish and later to establish the conditions and the type of correspondence between them. The obtained results will shed light on the problem that arises from the difference between the quantifiers that belong to markers and that can affect the teaching and acquisition of both languages on the one hand, and the translation in both directions on the other.

Key words: discourse marker, reformulation, distancing or separation, quantifier.

\section{Introducción}

El estudio de los marcadores discursivos (a continuación MD) pertenece al campo de las investigaciones suprasintácticas o discursivas, por lo que su desarrollo corre paralelamente con el desarrollo de la pragmática. Esta, a su vez, gira en torno a la noción de usuario y se ocupa de dilucidar cómo utilizamos el complejo sistema de los dispositivos lingüísticos de una determinada lengua en el proceso de interacción comunicativa entre el emisor y el receptor para decir más de lo que está dicho. Esto nos lleva a las raíces filosóficas de la disciplina y a sus primeros constructores.

El filósofo John L. Austin cuestionó el enfoque de la lengua vía condiciones de verdad y concibió la teoría tripartita de los actos de habla que engloba los actos locutivo (decir algo), ilocutivo (utilizar lo dicho) y perlocutivo (provocar resultado con lo dicho). A otro filósofo británico, Herbert Paul Grice, se deben los conceptos clave de la ostensión y la inferencia. Cualquier acto de comunicación es ostensivo, sea verbalmente realizado o no; en ambos casos se deriva o se infiere algún sentido en una situación dada. De ahí otro término central en la pragmática, el del contexto, que sugiere que no son importantes solo los sujetos participantes, sino también sus circunstancias, es decir, cuándo, cómo, dónde, por qué, etc. se efectúa el acto comunicativo.

Con estos postulados como base, Sperber y Wilson (1986 y 2006) desarrollaron su Teoría de la Pertinencia ${ }^{2}$ (TP) con dos principios fundamentales. El primero es de corte cognitivo: "Human cognition tends to be geared to the maximization of relevance", $y$ el segundo de carácter comunicativo: "Every ostensive stimulus conveys a presumption of its own optimal relevance" (Sperber y Wilson 2006: 610-612). Para estos autores el contexto "is a psychological construct, a subset of the hearer's assumptions about the world" (Sperber y Wilson 1986: 15), por lo que inferir no es lo mismo que deducir. La deducción pertenece a la esfera de la lógica, mientras que la inferencia tiene que ver con actitudes y creencias. De ahí deriva la diferencia entre significado, propio de las formas lógicas semánticamente completas, y sentido, propio de las formas semánticamente incompletas

2 Muchos autores españoles han adoptado el término inglés relevance; Portolés en todos sus trabajos insiste en que la única traducción correcta es Teoría de la Pertinencia.

BEOIBERÍsTICA Vol. II / Número 1 (2018) | 57-72 
y realizado en un concreto contexto. Por tanto, el significado es fijo y los sentidos varían según las situaciones.

En esta linea sigue otra dicotomía esencial de la que se vale la pragmática, la que desarrolló Blakemore (1987), la distinción entre significado conceptual y significado procedimental (o de procesamiento). El primero es denotativo y el segundo instructivo. Esto quiere decir que la función de los MD es instruir las inferencias o, en palabras de Fraser (1996: 168), se trata de alinguistically encoded clues which signal the speaker's potential communicative intentions".

Finalmente, otra teoría relevante para los estudios pragmáticos y los MD es la Teoría de la Argumentación (TA) de los lingüistas franceses Anscombre y Ducrot (1994) ${ }^{3}$, de carácter estrictamente lingüístico y dentro de la tradición estructuralista. El concepto esencial de la TA es el de orientación argumentativa cinterna de los enunciados hacia tal o cual tipo de conclusiones, orientación no deducible del contenido informativo" (ibidem: 55). El concepto de fuerza argumentativa conlleva la suposición de escalaridad. A partir de ella Portolés (1998) introduce el término de suficiencia argumentativa que se refiere al hecho de que en una situación dada algunos argumentos son suficientes para sacar unas conclusiones, pero no otras.

La TA se complementa e interactúa con otras dos teorías fundamentales, la Teoría Polifónica de la Enunciación y la Teoría de los Topoi desarrollada por Ducrot (1986 y 1988). Según la primera los argumentos están orientados hacia una determinada conclusión con ayuda del principio argumentativo de topos, "lugar común argumentativo" (Ducrot 1988: 63); los topoi en su gran parte son extralingüísticos, son nuestro contexto general. Respecto a la segunda, Ducrot (1986) reconoce que extiende libremente la teoría polifónica de Bajtín y concede al sentido del enunciado una pluralidad de voces.

\section{Marcadores de reformulación}

\subsection{Conceptos, definiciones, clasificación}

El concepto de marcador discursivo ha recibido distintas denominaciones. En el Diccionario de Análisis de Discurso (2005; a continuación DAD) se citan doce términos 4 , mientras que Cortés y Camacho (2005: 237) ofrecen una lista de los veinte utilizados en el ámbito hispano, incluyendo el de muletillas. Hemos optado por el de DM como el término más difundido entre los autores españoles cuyas aportaciones en este campo son

3 Utilizamos la traducción al castellano de esta obra de 1988 porque es una versión adaptada y actualizada por los propios autores para la edición española.

${ }^{4}$ Algunos de ellos son: apoyos del discurso, puntuadores, ligadores, conectores fáticos, marcadores de estructuración de la conversación, etc. 
más que considerables. Por la misma razón nos atenemos a la definición de Martín Zorraquino y Portolés Lázaro (1999: 4057) que siguió utilizando Portolés ([2001] 2011: 25-26):

Los 'marcadores del discurso' son unidades lingüísticas invariables, no ejercen una función sintáctica en el marco de la predicación oracional - son, pues, elementos marginales - y poseen un cometido coincidente en el discurso: el de guiar, de acuerdo con sus distintas propiedades morfosintácticas, semánticas y pragmáticas, las inferencias que se realizan en la comunicación.

Estos autores también han ofrecido la clasificación de los DM en cinco grupos de los que uno son los reformuladores. ${ }^{5}$

El término de reformulación fue introducido en la lingüística del texto alemana por Gülich y Kotschi (1983). Es curioso que las entradas 'reformular' y 'reformulación' no consten en el Diccionario de la lengua española de la Real Academia (2014) y tampoco en el Diccionario del español actual (1999), no obstante, su significado es fácil de deducir basándose en los elementales conocimientos de morfología española.

Lo primero a lo que la reformulación asocia es a la repetición. La repetición en la lengua se estudia en diferentes niveles gramaticales y en la literatura como recurso estilístico y figura retórica. A finales de los 80 empezó a estudiarse dentro de la pragmática como fenómeno sui generis. Desde la perspectiva cognitivista, Blakmore (1993) escribe sobre la reformulación como un dispositivo para alcanzar la pertinencia óptima y efectos contextuales que varían en función del tipo textual en el que se utilizan. Bazzanella (1996) observa que lo repetido deja de ser lo mismo en los niveles semántico y pragmático y Camacho Adarve (2009) hace hincapié en el mismo hecho, por todo lo cual llega a considerar que "no hay que rechazar la posibilidad de repetición como quasimarcador discursivo" (2009: 36).

Portolés (2011: 105 y 141) define los reformuladores como "marcadores que presentan el miembro del discurso en el que se encuentran como nueva formulación de lo que se pretendió decir con un miembro anterior." Como se ve, el autor introduce el término de miembro discursivo, un instrumento de precisión analítica, definiéndolo como "unidad lingüística mínima en la que se puede localizar un marcador [y que] puede ser menor que un enunciado" (ibidem 40-41).

Existen diferentes clasificaciones de diferentes autores, pero aquí nos atendremos a dos, casi coincidentes. Una es de Martín Zorraquino y Portolés (1999: 4082) que dividen todos los reformuladores en cuatro grupos:

- explicativos (o sea, es decir, esto es, etc.)

- recapitualtivos (en suma, en conclusión, en fin, etc.)

- rectificativos (mejor dicho, más bien, etc.) y

- de distanciamiento (en cualquier caso, en todo caso, etc.).

${ }^{5}$ Los cuatro grupos restantes son: estructuradores de la información, conectores, operadores argumentativos o discursivos y marcadores conversacionales o de control de contacto (CDLE 1999: 40814082 y Portolés 2011: 146).

BEOIBERÍsTICA Vol. II / Número 1 (2018) | 57-72 
La otra es de Garcés Gómez (2007: 532-533 y 2008: 87), la autora que más ha tratado el tema de la reformulación y que distingue cinco grupos, ya que ha refinado la clase de recapitulativos separando de ellos los marcadores de reconsideración (en definitiva, a fin de cuentas, total, etc.).

En la serbística y croatística es difícil hablar de una tipología muy específica, ya que los estudios discursivos todavía están bastante desatendidos. Merece la pena mencionar las obras precursoras de Velčić (1987) y de Stevović (1958: 160-162) quien dentro del conjunto "modalne reči i izrazi" (palabras y expresiones modales) enumera varias funciones de estas unidades como aclaración, intensificación, limitación, precisión, etc. Ristić (2009) se centra principalmente en los modificadores léxicos y su análisis se detiene en el nivel sintagmático, aunque hable de funciones discursivas. Aún así, sus listas son aprovechables para extraer algunos marcadores de reformulación.

\subsection{Marcadores reformulativos de distanciamiento}

Los marcadores de distanciamiento, o de separación, como los denomina Garcés Gómez (2008) upresentan expresamente como no relevante un miembro del discurso anterior a aquel que los acoge" (Martín Zorraquino y Portolés Lázaro 1999: 4128). Cuenca (2010: 69) los clasifica como conectores parentéticos ${ }^{6}$ de contraste y los sitúa en el grupo de concesivos, junto con, por ejemplo, no obstante, a pesar de todo, al fin y al cabo y total. Para Fuentes Rodríguez (1998) también son concesivos y Casado Velarde (2006 y 2008) menciona en todo caso en función de atenuante.

La característica común de este conjunto es que todos los marcadores contienen cuantificadores (todo, todos y cualquiera) que condicionan su significado $\mathrm{y}$, en consecuencia, las inferencias que instruyen. Los tres son cuantificadores universales "que denotan la totalidad de los valores que puede tomar la expresión cuantificada" (Sánchez López 1999: 1037). La cuantificación de todo denota globalidad y "Como parte de un conector [...] se aplica sobre un conjunto de situaciones posibles realizadas en la estructura sintáctica bajo la forma de todos los argumentos previos a la aparición del conector" (Rodríguez Ramalle 2005: 78). La de todos y cualquiera no es generalizadora, sino atomizadora, se refiere a los miembros contables de un conjunto dado; cualquiera se caracteriza por el matiz semántico de indiferencia.

En serbio el significado de todo lo tiene el adjetivo sav, y el de todos y cualquiera, svaki; el ingrediente de indiferencia se consigue con bilo koji / ikoji (quien / lo que quiera que sea). Este último junto con sav no participa en la construcción de los marcadores de distanciamiento. Las diferencias semánticas de las estructuras que las soportan (pero no las

\footnotetext{
${ }^{6}$ Los MD parentéticos son los que van entre pausas o comas.
} 
estructuras en sí) siempre señalizan la fuente de posibles dificultades, tanto en la adquisición, como en la traducción.

En la siguiente tabla los enumeramos y a continuación proseguimos con el análisis de cada uno de ellos.

\begin{tabular}{|l|l|}
\hline \multicolumn{2}{|c|}{ Marcadores reformulativos de distanciamiento o separación } \\
\hline español & serbio \\
\hline en todo caso & u svakom slučaju \\
en cualquier caso & u najboljem slučaju \\
de todos modos & u najmanju ruku \\
de todas formas/maneras & na svaki način \\
de cualquier modo/forma/manera & \\
\hline
\end{tabular}

\subsubsection{En todo caso}

Como el resto de los marcadores, en todo caso también instruye varias inferencias. En el Diccionario de Partículas (DP) se define como locución adverbial omniconcesiva y en Diccionario de Conectores y Operadores del Español (DCOE) como conector concesivo y de oposición, que en Fuentes Rodríguez (1998: 35) se explicó así: "a pesar de lo que he dicho en el primero, hay que decir lo segundo". Por tanto se trata de una invalidación del primer miembro y su sustitución con el nuevo, como en (1), donde el cuantificador abarca todas las novelas a la vez. Pero como se trata de unidades contables, en la traducción se puede utilizar u svakom slučaju, literalmente en cada uno de los casos; por otro lado, si el plural novelas se tomara en conjunto, el marcador también se podría traducir con $u$ celini gledano (en genera), que es un operador ${ }^{7}$ enunciativo, pero con el que se pierde el significado de distanciamiento:

(1) "La ciencia", prosigue Barceló, "se hace en los laboratorios y en las revistas especializadas, no en las novelas. [...]" No se trata, en todo caso, de novelas de divulgación científica, asegura Javier Ordóñez «igual que Frankenstein no se escribió para explicar el efecto del galvanismo en los tejidos, sino que era una reflexión moral sobre un mito que sigue funcionando". (El País. Babelia, 04/10/2003, CREA)

("Naukom se" nastavio je Barselo, "bave u laboratorijama y specijalizovanim časopisima, ne u romanima". U svakom slučaju, nije reč o romanima popularne nauke, tvrdi Havijer Ordonjes “isto kao što Frankenštajn nije napisan da objasni uticaj galvanizacije na tkiva, nego je bio etičko razmišljanje o mitu koji i dalje funkcionišem.)

${ }^{7}$ Los operadores son un tipo de MD que modifican únicamente el miembro del discurso en el que se encuentran.

BEOIBERÍsTICA Vol. II / Número 1 (2018) | 57-72 
Sin embargo, con este marcador se necesita prestar especial atención a varios factores, no solo a la gradación de los argumentos. Esto se refleja en diferentes posibilidades de traducción, para lo que acudimos al análisis de Portolés (1998: 213-216), quien se centra en la suficiencia argumentativa y la polifonía:

(2)[...] de aquel grupo de intelectuales que velaron sus armas literarias en los años de la República, sólo permanecieron en España los que apoyaron al bando de los vencedores o los que, en todo caso, no apoyaron abiertamente al de los vencidos. (Anales de Literatura Española, no 14, 2001, CREA)

([...] od grupe intelektualaca koji su pritajili svoje književno oružje u godinama Republike, u Španiji su ostali samo oni koji su podržali pobedničku stranu ili oni koji (u najmanju ruku) bar nisu otvoreno podržavali poraženu.)

(3)[...] gracias a la impagable colaboración de su hija, Cecilia, pude seleccionar, a veces traducir $\mathrm{y}$, en todo caso, comentar una enorme serie de "Escritos" originales del admirado maestro... (ABC Cultural, 22/11/1996, CREA)

([...] zahvaljujući neprocenjivoj saradnji njegove ćerke Sesilije, mogao sam da odberem, ponekad da prevedem, ili bar da tumačim ogromnu količinu originalnih "Spisa" poštovanog učitelja.)

No apoyar abiertamente a los vencidos es un argumento más débil que apoyar a los vencedores, pero como el locutor es el mismo la suficiencia argumentativa se considera satisfecha. Igualmente en el (3), comentar es menos que traducir, que supone la totalidad del documento o de la documentación, pero para el locutor es suficiente. Como se ve, en la traducción serbia esta condición se refleja en la traducción en la que ya no se puede utilizar u svakom slučaju, sino u najmanju ruku, o la partícula sinónima bar en combinación con la conjunción disyuntiva ili, ambas equivalen a "como poco" en la paráfrasis de Portolés (1998: 215).

Otro efecto de sentido derivado de la suficiencia argumentativa es cuando se expresan reservas respecto del contenido del miembro que precede al marcador, cuando este "modaliza[r] una afirmación anterior restándole certidumbre" (Martín Zorraquino y Portolés Lázaro 1999: 4131), o, añadiríamos, restándole fuerza al situar el argumento en una posición más baja en la escala argumentativa:

(4) Okolo naokolo, po groblju, [...] rasejani su grobovi onih što nekada behu vezani za gospa Nolu, ili za njenog muža, ili bar za imanje njenog muža. (SrpCorp2013, ej. no 97)

(Por todo el alrededor, por el cementerio, están esparcidas las tumbas de los que antaño se relacionaban con la señora Nola, o con su marido, o en todo caso con la finca de su marido.) 
Sin embargo, cuando en todo caso introduce un argumento como concesión al interlocutor, ya no se cumple la suficiencia argumentativa, y la traducción al serbio aprovecha una tercera opción:

(5) No se veía nada, en todo caso se intuía o se creía. Seguía siendo cuestión de fe.

(El Mundo, 28/07/1994, CREA)

(Nije se videlo ništa, u najboljem slučaju, naslućivalo se ili se verovalo. I dalje je to bilo pitanje vere.)

Según la explicación de Portolés (ibidem: 214), como el miembro introducido por el marcador se sitúa en el extremo más alto de la escala argumentativa, ya que no se podía más que intuir o creer, en todo caso se interpreta como una concesión al interlocutor. En serbio esto se registra con el uso del marcador u najboljem slučaju que equivale a "como mucho".

Teniendo en cuenta que el cuantificador todo se aplica a conjuntos o, como apunta Rodríguez Ramalle (2005: 86) a "una parte mayor dentro de un conjunto", la exclusión del miembro anterior que es negado implica el uso de la conjunción sino:

(6) No se cita en ningún momento el concepto de "ataque preventivo" usado por EE UU, sino en todo caso, acción preventiva" o "compromiso preventivo", y no se refiere sólo a aspectos militares, aunque los incluye. (El País, 20/06/2003, CREA)

( $\mathrm{Ni}$ jednog trenutka se ne pominje pojam "preventivnog napada" koji koriste SAD, nego u najboljem slučaju "preventivna akcija" ili "preventivno angažovanje", a ne odnosi se samo na vojne aspekte, mada ih uključuje.)

En el ejemplo citado no es necesario inferir un interlocutor, ya que explícitamente se citan palabras ajenas. La exclusión es casi completa ya que el concepto de ataque se sustituye por completo, solo permanece el determinante "preventivo".

Para resumir, el marcador de distanciamiento o separación en todo caso tiene tres posibles traducciones al serbio porque no coincide el uso del los cuantificadores en las dos lenguas. Una es con u svakom slučaju cuando el cuantificador se refiere a la anulación de un conjunto compuesto por elementos contables. Otra es con u najmanju ruku cuando hay solo un locutor y se cumple la condición de la suficiencia argumentativa. Y la tercera, u najboljem slučaju refleja la concesión al interlocutor, porque se mantiene que la suficiencia argumentativa no se ha conseguido.

\subsubsection{En cualquier caso}

El cuantificador que contiene en cualquier caso, recordamos, hace referencia a entidades particulares y contables, por lo que el marcador suele encontrarse en las estructuras cuyo primer miembro discursivo es complejo. En el $D P, D C O E$ y en Fuentes Rodríguez (1998: 35) se determina como concesivo, igual que en todo caso. Pero más que 
dar concesiones, su función es invalidar los argumentos precedentes favoreciendo el que se introduce en su segmento. Martín Zorraquino y Portolés (1999: 4129) y Portolés (1998: 213, n. 16 y 2011: 123) señalan que el tópico de los dos segmentos es diferente, por lo que no se combina con la conjunción $\sin 0^{8}$, lo que diferencia este marcador de en todo caso.

Dada la pluralidad de elementos en el primer miembro discursivo y la referencia del marcador a cada uno de ellos, la verdadera correspondencia en serbio es con $u$ svakom slučaju, que en este caso no supone condiciones especiales por el carácter del cuantificador.

En el ejemplo que sigue, en el que, además, la posición final del marcador no es muy frecuente, el miembro que antecede contiene dos condiciones, la piel clara y la facilidad de ruborizarse, y el marcador instruye la eliminación de ambas introduciendo un nuevo tópico (hay que olvidar los colores en cuestión); en este caso puntual la traducción se adapta al contexto y no exige un marcador:

(7) [...] son colores más difíciles de llevar a la luz del día; sin embargo, son ideales de noche. Si tienes la piel muy clara y te ruborizas fácilmente, olvídate de ellos, en cualquier caso. ( Telva, 12/1997, CREA)

([...] to su boje koje je teže nositi na dnevnom svetlu; međutim, idealne su za noć.

Ako ti je koža vrlo svetla i lako pocrveniš, zaboravi na njih, u oba slučaja.)

En (8) el primer segmento es implícito, puesto que el miembro con el marcador inicia un subcapítulo. En consecuencia, se infiere que en cualquier caso abarca toda la complejidad contada anteriormente y que, de hecho, no se trata de alternativas. Entonces, en ejemplos como este es posible la conmutación ${ }^{9}$ con en todo caso:

(8) Es preciso señalar que, en cualquier caso, [...] el cuadro continuará siendo tan complejo quizás como el que queda expuesto para el siglo XVII. (Hispania Nova.

Revista de Historia Contemporánea, n 3, 2003)

Es preciso señalar que, en todo caso, el cuadro continuará siendo...

(Treba reći da će slika u svakom slučaju i dalje biti možda isto tako složena kao što je to objašnjeno za XVII vek.)

Lo mismo ocurre en (9) que también puede recibir una lectura abarcadora en vez de particularizada como en el original; esto es posible porque el valor nominal de los

${ }^{8}$ Fernández Rodríguez (2009), sin embargo, en la entrada correspondiente del DCOE, apunta que "es frecuente con pero, sino, $y$, a", pero no ofrece ningún ejemplo con sino.

${ }^{9}$ El método de conmutación se aplica en el análisis semántico de los MD y según Portolés (2011: 79) consiste en agrupar los marcadores que se pueden intercambiar en un contexto dado, pero también en encontrar aquellos contextos en los que tal sustitución no sería posible. 
números mencionados no importa ya que el adjetivo "demasiados" los engloba todos. La traducción no cambia ni en el ejemplo anterior, ni en este:

(9) ¿Cuántos poetas, nacidos tras la guerra civil, se dieron a conocer desde mediados de los años 60? ¿Quinientos? ¿Mil? ¿Cinco mil? Demasiados, en cualquier caso. $Y$ al menos ciento cincuenta de esos poetas (hice la lista) publicaron en colecciones de renombre... ( $A B C$ Cultural, 20/12/1996, CREA)

...Demasiados, en todo caso.

(Koliko je pesnika rođenih posle građanskog rata postalo poznato sredinom šezdesetih? Petsto? Hiljadu? Pet hiljada? Previše ih je, u svakom slučaju. A najmanje sto pedest tih pesnika (sastavio sam spisak) objavljivali su u poznatim bibliotekama...)

En (10) y (11) el marcador no anula ni debilita las alternativas expuestas, sino que introduciendo otra opción las tacha de irrelevantes:

(10) Ne znam da li ću ti ikad više pisati. Mislila sam da je to odlična ideja, ali sad više nisam tako sigurna. U svakom slučaju, žao mi je što si tako star i bolestan i što se nismo bolje upoznali onda kad je prilika za to zaista postojala. (SrpCorp2013, ej. $\mathrm{n}^{\circ}$ 98)

(No sé si volveré a escribirte jamás. Pensaba que era una idea excelente, pero ya no estoy tan segura. En cualquier caso, siento porque eres tan viejo y estás tan enfermo y porque no nos conocimos mejor cuando para esto realmente existió una oportunidad.)

(11) I onda, kada sam razgrnula dve stabljike, videla sam puža. Videla sam običnog puža kako me posmatra. Ne umem da kažem kako me je posmatrao, ne znam kako da opišem pogled puža. Da li je to bio prezir ili mržnja. U svakom slučaju, bilo je nečeg neprijateljskog u tom pogledu. (SrpCorp2013, ej. $\mathrm{n}^{\circ}$ 107)

( $Y$ entonces, al separar dos tallos, vi al caracol. Vi a un caracol ordinario mirándome. No sé decir cómo me miraba, no sé cómo describir la mirada de un caracol. Si era de desprecio o de odio. En cualqueir caso, en esa mirada había algo hostil.)

Para un hablante serbio sin duda es más fácil la adquisición del marcador en cualquier caso que del en todo caso. La razón deriva en primer lugar de su componente cuantificadora, y en el segundo del matiz de indefinitud característico de cualquier que lo acerca a todo. 
2.2.3. De todos modos, de todas formas, de todas maneras, de cualquier modo, de cualquier forma, de cualquier manera

Los marcadores de este conjunto son sinónimos que en el $D C O E$, en el que se encuentran los primeros tres, se etiquetan como concesivos, y en el $D P$ como locuciones omniconcesivas. La definición del Diccionario de Partículas discursivas del español (DPDE), que también se refiere a los primeros tres (porque los otros son menos frecuentes), es aplicable a todos: “Presenta[n] el miembro del discurso en el que aparece[n] como más pertinente para la continuación del discurso que otras opciones anteriores, tanto explícitas como sobreentendidas". De esto se concluye, como señalan Martín Zorraquino y Portolés Lázaro (1999: 4132), que siempre comentan tópicos diferentes, por lo que no se combinan con la conjunción sino. A su vez, Garcés Gómez (2008: 143) distingue entre dos grupos de casos:

- el marcador instruye la supresión de la pertinencia del miembro de referencia;

- el marcador instruye que los argumentos precedentes no ejercen influencia sobre la conclusión final del acto de reformulación.

Para todos estos marcadores en serbio hay uno, na svaki način, pero es bastante menos frecuente que u svakom slučaju, que sirve perfectamente para la traducción también en estos casos.

Empezamos con los ejemplos del primer grupo que ilustran la privación de la pertinencia del primer miembro:

(12) De niña adoraba el verano. Luego lo detesté, por razones múltiples y avatares biográficos [...]. Pero entonces, hablo de 1987, la palabra verano no suscitaba en mí asociaciones fatídicas de ninguna clase. $Y$ de todos modos, no era verano allá, en esa playa del Pacífico, al otro lado del mundo. (El Mundo, 23/08/1996, CREA) (Kao devojčica obožavala sam leto. Posle sam ga mrzela, iz više razloga i nemilih biografskih događaja. Ali tada, govorim o 1987, reč leto u meni nije budila nikakve zlokobne asocijacije. A u svakom slučaju, tamo, na toj pacifičkoj plaži, na drugoj strani sveta, i nije bilo leto.)

(13) - A koliki su prihodi u zemlji, gospodine ministre? To je, držim, važna stvar ? - The, to baš i nije važno! ... Kako da vam kažem? Upravo, ne zna se još koliki su prihodi. Čitao sam nešto o tome u jednom stranom listu, ali ko zna je li to tačno? Tek, na svaki način, ima dosta prihoda, dosta, bez sumnje! (SrpCorp2013, ej. no 33)

(-Y icuál es la renta del país, señor ministro? Esto, creo, es un asunto importante.

- En fin, ieso no es tan importante! ... A ver, icómo se lo digo? De hecho, todavía no se sabe la cuantía de la renta. Leí algo en un periódico extranjero, pero quién sabrá si es verdad. De todos modos, ies bastante alta la renta, alta, sin duda!) 
En los siguientes ejemplos las inferencias que se sacan a partir del primer miembro conducirían a la conclusión contraria a la que instruye el marcador; en el primero de ellos, esta oposición va corroborada con la conjunción pero:

(14) Yo estoy ansioso por conseguir resultados, y a mi empresa la manejo con objetivos y plazos. Pero, en ALAF no puedo determinar el objetivo y el tiempo que puede tardar en conseguir, porque intervienen otros factores. Pero, de todas formas, lo acepto con hidalguía. (Vía Libre, no 459, 12/2002) (Željno očekujem postizanje rezultata, i svoje preduzeće vodim ciljano i s rokovima. Ali u ALAF-u ne mogu da odredim ciljeve i vreme koje mi je potrebno da ih dostignem jer se tu javalju drugi činioci. Ali, u svakom slučaju, viteški prihvatam.)

(15) En cuanto al castellano, con sólo analizar la nula influencia de Hispanoamérica dentro del contexto mundial entenderemos el porqué de la menor difusión mundial de dicho idioma. Reconozcamos, de todas maneras, que nuestra reciente democracia empieza a dar sus frutos en este campo, ya que tanto en Estados Unidos como en Japón y otros países está de moda lo español... ( $L a$ Vanguardia, 02/02/1994, CREA)

(Što se tiče kastiljanskog, ako samo analiziramo nikakav uticaj hispanske Amerike u svetskim okvirima, razuemećemo razlog manje svetske rasprostranjenosti pomenutog jezika. $\mathrm{Na}$ svaki način/U svakom slučaju, treba priznati da naša mlada demokratija počinje da daje plodove na ovom polju pošto je u SAD kao i u Japanu i drugim zemljama u modi sve što je špansko.)

En el segundo grupo, reiteramos, están los casos en los que uno o más argumentos previamente expuestos se presentan como insuficientes o irrelevantes:

(16) Se define como liberal en todo [Torrente Ballester], incluido el lenguaje, cuando se le inquiere sobre la salud del idioma español. "Yo, en realidad" señala sin perder su habitual retranca uescribo en ferrolano. En castellano escriben los de Valladolid, pero de todos modos no me opongo al uso de palabras extranjeras siempre que no exista un equivalente en nuestra lenguan. (El País, 08/05/1997, CREA)

(Kad ga pitamo o zdravlju španskog, određuje se kao liberalan u svemu, pa i u jeziku. "Ja u stvari", kaže ne gubeći svoju uobičajenu prepredenost, "pišem na ferolskom. Na kastiljanskom pišu oni iz Valjadolida, ali u svakom slučaju, nemam ništa protiv stranih reči ako u našem jeziku nema odgovarajućem.)

(17) Sve je to istorija... jedna istorija francuske književnosti, već neko vreme ultrapolitizirana... Nije to naša tema. Na svaki način, vidite: judaizam u mojim knjigama je pre igra slučaja i sudbine.

(SrpCorp2013, ej. no 97) 
(Todo esto es historia... una historia de la literatura francesa, desde hace algún tiempo ultrapolitizada... No es este nuestro tema. De todos modos, mire: el judaísmo en mis libros es más un juego del azar y del destino.)

Si todos los marcadores citados en este apartado no son problemáticos para un hablante serbio, es fácil que sin una adecuada instrucción se le escape la sutil diferencia entre en cualquier caso y de todos modos (o de todas formas/maneras) por la mencionada diferencia semántica entre los cuantificadores: la particularización de cualquier que no tienen los otros. Como observan Martín Zorraquino y Portolés Lázaro (1999: 4132), si se trata de alternativas que se invalidan y no influyen en la conclusión, se usa en cualquier caso (como en 7), pero si el argumento que le precede se suprime, son preferibles estos otros (como en 12 y 13).

Para reflejar esta diferencia en la traducción se debe acudir a otras soluciones, si no, utilizando u svakom slučaju se pierde una parte del sentido:

(18) Hijo: Esta vez me portaré bien.

Padre: (a) En cualquier caso, tú te vas inmediatamente a la cama.

1999: 4133)

(b) De todas formas, tú te vas inmediatamente a la cama. (ibidem,

(Dete: Biću dobar ovoga puta.

Roditelj: Kako god (hoćeš), smesta ideš u krevet.

U svakom slučaju odmah ideš u krevet.)

El significado de kako god es como sea, sea como fuere, así que se conserva la alternativa del original. Para resaltar la duda en la promesa del niño se puede introducir el verbo volitivo hteti (querer) con el resultado de como quieras que no suprime la alternativa entre portarse bien o no. Los marcadores del grupo de de todas formas no suscitan duda, mandan el mensaje de que el argumento, la promesa del niño no sirve, por lo que el marcador u svakom slučaju es conveniente.

Gracias a la sinonimia de palabras modo, forma y manera, así como a dos cuantificadores que intervienen en este tipo de reformulación, el español dispone de seis marcadores a los que en serbio corresponden dos, ambos con un mismo cuantificador svaki. En cuanto a la adquisición y la traducción, estos seis no presentan problemas porque su significado es claro.

\section{Conclusiones}

Buscando diferentes efectos de sentido y el entorno contextual que los condicionaba, hemos procedido al análisis contrastivo de los MD de distanciamiento o separacićon dentro de fragmentos de discursos en español y en serbio. Con este enfoque 
hemos conseguido, primero, determinar los marcadores de distanciamiento o separación en serbio, que son los siguientes: u svakom slučaju, u najboljem slučaju, u najmanju ruku $i$ na svaki način.

En segundo lugar, los hemos clasificado según los criterios desarollados por los pragmáticos hispanistas a fin de unificar el análisis. Puesto que estos criterios no son los que se aplican en la serbística (ni en la croatística), la clasificación es nueva y, además, contiene unidades que en la bibliografía no han sido tratadas como marcadores discursivos.

En tercer lugar, hemos prestado atención a los cuantificadores contenidos en los marcadores de este grupo, que no coinciden en las dos lenguas. Como siempre, las diferencias presentan problemas tanto en la enseñanza y la adquisición, como en la traducción en ambas direcciones. Una vez detectados, dichos problemas se pueden evitar aplicando la instrucción adecuada.

Finalmente, hemos establecido las siguientes correspondencias:

- En todo caso puede equivaler a u svakom slučaju, u najmanju ruku, ili bar, así como a u najboljem slučaju, dependiendo de la suficiencia argumentativa y la relación que existe entre el locutor e interlocutor.

- En cualquier caso siempre se corresponde con u svakom slučaju debido a la coincidencia del cuantificador.

- Para de todos modos, de todas formas, de todas maneras, de cualquier modo, de cualquier forma, de cualquier manera en serbio se utilizará de nuevo u svakom slučaju, aunque también se puede contar con el menos frecuente na svaki način.

En todas las elecciones, siempre que haya más de una opción, la decisión se tomará para cada contexto particular.

\section{BIBLIOGRAFÍA}

Austin, John L. Cómo hacer cosas con palabras. Barcelona: Ediciones Paidos, 1990. Impreso.

Anscombre, Jean-Claude y Oswald Ducrot. La argumentación en la lengua. Madrid: Gredos, 1994 [1988]. Impreso.

Bazzanella, C. (ed.). Repetition in dialogue. Tübingen: Max Niemayer Verlag, 1996. Print. Blakemore, Diane. Semantic constraints on relevance. Oxford: Blackwell, 1987. Print.

—. "The relevance of reformulations." Language and Literature 2.2. (1993): 101-120. Print.

Briz, Antonio el al. (coords.). Diccionario de partículas discursivas del español. Web. 15 Oct. 2017.

Camacho Adarve, Ma Matilde. Análisis del discurso y repetición: plabras, actitudes y sentimientos. Madrid: Arco / Libros (Oralia, Anejos 5), 2009. Impreso.

Casado Velarde, Manuel. Introducción a la gramática del texto del español. Madrid: Arco / Libros, 2006. Impreso. 
-. "Lingüística del texto y marcadores del discurso". M. a Antonia Martín Zorraquino y Estrella Montolío Durán (coords.), Los marcadores del discurso. Teoría y análisis. Madrid: Arco / Libros. 2008: 55-70. Impreso.

Cortés, Luis y $M^{a}$ Matilde Camacho. Unidades de segmentación y marcadores del discurso. Madrid: Arco / Libros, 2005. Impreso.

Cuenca, Ma Josep. Gramática del texto. Madrid: Arco / Libros, 2010. Impreso.

Charaudeau, Patrick y Dominique Maingueneau (dir.). Diccionario de análisis del discurso (DAD). Buenos Aires - Madrid: Amorrortu Editores, 2005. Impreso.

Ducrot, Oswald. El decir y lo dicho. Barcelona, Buenos Aires, México: Paidós, 1986. Impreso.

-. "Argumentación y 'topoi' argumentativos". Lenguaje en contexto 1 (1988): 63-84. Impreso.

Fraser, Bruce. «Pragmatic Markers». Pragmatics 6:2 (1996): 167-190. Print.

Fuentes Rodríguez, Catalina. La sintaxis de los relacionantes supraoracionales. Madrid: Arco / Libros, 1998. Impreso.

-. Diccionario de conectores y operadores del español (DCOE). Madrid: Arco / Libros, 2009. Impreso.

Gülich, Elizabeth y Thomas Kotschi. 1983. "Les marquers de reformulation paraphrastique". Cahiers de Linguistique Française, 5 (1983): 305-351. Imprimé.

Garcés Gómez, Ma Pilar. "La reformulación parafrástica en el discurso oral (español)". Luis Cortés Rodríguez et al. (eds.), Discurso y Oralidad. Homenaje al profesor José Jesús de Bustos Tovar. Madrid: Arco / Libros (Oralia, Anejos 3/2), 2007: 529-542. Impreso.

- La organización del discurso: marcadores de ordenación y de reformulación. Madrid / Frankfurt am Main: Iberoamericana / Vervuert, 2008. Impreso.

Grice, Herbert Paul. "Utterer's Meaning and Intention". The Philosophical Review, 78/2 (1969): 147-177. Print.

Grice, Herbert Paul. "Logic and Conversation". Peter Cole, et al. Syntax and Semantics 3: Speech Acts (1975): 41-58. Print.

Martín Zorraquino, Ma Antonia y José Portolés Lázaro. "Los marcadores del discurso". Ignacio Bosque y Violeta Demonte (dirs.), Gramática Descriptiva de la Lengua Española, I-IIl. Madrid: Espasa-Calpe, 1999: 4051-4213. Impreso.

Matematički fakultet Univerziteta u Beogradu. Korpus savremenog srpskog jezika SrpKor2013. 2013. Web. 03-15 Oct. 2017.

Portolés, Jose. "El concepto de suficiencia argumentativa”. Signo y seña, 9 (1998): 199 224. Impreso.

-. Marcadores del discurso, 4⿳亠丷 ed. Madrid: Ariel, 2011. Impreso.

Real Academia Española. Corpus de Referencia del Español Actual (CREA). 2008. Web. 05-15 Oct. 2017. 
—. Diccionario de la lengua española (Actualización 2017), (DRAE). Madrid: Espasa. Web. 12-17 Oct. 2017.

Ristić, Stana. Modifikacija značenja i leksički modifikatori u srpskom jeziku. Beograd: Institut za srpski jezik SANU, 2009. Štampano.

Rodríguez Ramalle, Teresa $M^{a}$. "Los conectores entre la sintaxis, la semántica y la pragmática”. CLAC 24 (2005): 74-90. Web. 16 Oct. 2017.

Sánchez López, C. "Los cuantificadores: clases de cuantificadores y estructuras cuantificativas". Ignacio Bosque y Violeta Demonte (dirs.), Gramática Descriptiva de la Lengua Española, I-IIl. Madrid: Espasa-Calpe, 1999: 1025-1128. Impreso.

Santos Río, Luis. Diccionario de partículas. Salamanca: Luso-Española de Ediciones, 2003. Impreso.

Sperber, Dan y Deirdre Wilson. Relevance. Communication and Cognition. Oxford: Basil Blackwell, 1986. Print.

-. "Relevance Theory". Larry Horn and Gregory Ward (eds.), The handbook of Pragmatics. Oxford: Blackwell, 2006: 607-632. Print.

Stevović, Igrutin. Funkcionalna gramatika srpskohrvatskog jezika. Sarajevo: Svjetlost, 1958. Štampano.

Velčić, Mirna. Uvod u lingvistiku teksta. Zagreb: Školska knjiga, 1987. Štampano.

Fecha de recepción: 15 de enero de 2018. Fecha de aceptación: 12 de abril de 2018. 